Michael Custodis (Münster)

\title{
Replik auf die Stellungnahme von Albrecht Dümling
}

Wie ich in meinem im ersten Heft des 65. Jahrgangs der Musikforschung erschienenen Aufsatz zu zeigen versuchte, fungierte Friedrich Blume vor und nach 1945 als mächtiger Repräsentant der deutschen Musikwissenschaft. Von zentraler strategischer Bedeutung für seine Karriere im NS-Staat und das anschließende Spruchkammerverfahren erwiesen sich seine Einlassungen zum Thema „Musik und Rasse“. Dieser Interpretation widerspricht Albrecht Dümling, so dass ich meine Position noch einmal präzisieren und bekräftigen möchte, da ich sie für eine bisweilen vielleicht unbequeme, aber unausweichliche kritische Betrachtung der musikwissenschaftlichen Fachgeschichte für wichtig halte.

In Archiven neu entdeckte Materialien sind zunächst einmal zu interpretierende Tatsachen, mit denen sich bisherige Sichtweisen bestätigen, ergänzen oder korrigieren lassen. Wenn man aber mit der Faktenlage mehrerer hundert Seiten Quellenmaterials und bereits publizierter Dokumente zu Blume ${ }^{1}$ offensichtlich nicht vertraut ist, sind Widersprüche zu bisherigen Ansichten nicht unbedingt den neuen, archivgestützten Schlussfolgerungen anzulasten. Reduziert man Blumes Auseinandersetzung mit dem Thema „Musik und Rasse“ auf seinen entsprechenden Vortrag bei den Reichsmusiktagen 1938, in deren Rahmen auch die von Dümling verdienstvoll rekonstruierte Propagandaschau Entartete Musik veranstaltet wurde, und verwendet Blumes eigene, nach 1945 vorgetragene Sichtweise als Deutungsmaßstab, so wie Dümling es tut, ohne erstens den strategischen Nutzen der in verschiedenen Varianten vorgelegten Einlassungen (Vortrag, Aufsatz und Buch) zu taxieren und zweitens deren systeminterne Rezeption vor 1945 zu berücksichtigen, wird man der Komplexität der Zusammenhänge in keiner Weise gerecht. Wie wenig Pauschalisierungen dabei weiterhelfen, lässt sich an einem kleinen Beispiel zeigen: An vielen Stellen der Personalakte ist nachweisbar, dass Blume sich routiniert der standardisierten Grußformel „Heil Hitler!“ bediente, was entsprechend den formalen Vorgaben für amtliche Dokumente nicht überrascht. Dass er aber auch in seiner privaten Korrespondenz ohne äußere Zwänge mit dieser Formel zeichnete, ist nachprüfbar an jenem (seit zwei Jahren publizierten) Brief vom 7. November $1938^{2}$, mit dem er seinen ersten Doktoranden Wolfgang Steinecke im Zuge seiner mit Ministerialrat Herman-Walther Frey geführten Berufungsverhandlungen unter Vernachlässigung jeglicher Wissenschaftlichkeit zur Habilitation drängte.

Über Blumes tatsächliche politische Einstellung lassen die gefundenen Archivalien keine Aussagen zu, so dass sich meine Argumentation auf die strategische Planung seiner Karriere im NS-Staat und der Konsequenzen für die deutsche Nachkriegsmusikwissenschaft konzentrierte. Es wäre unrealistisch anzunehmen, während der NS-Herrschaft seien Universitätskarrieren gegen das System möglich gewesen. Dabei ist das Argument, Blume sei nicht Mitglied der NSDAP gewesen, noch kein Beweis einer oppositionellen Haltung, ebenso wenig wie ein Parteibuch ein pauschaler Beleg ideologischer Überzeugungen wäre. Denn zum einen hatte Blume bereits durch seine freiwillige Mitgliedschaft im

1 Michael Custodis, „Wolfgang Steinecke und die Gründung der Internationalen Ferienkurse“, in: Traditionen, Koalitionen, Visionen. Wolfgang Steinecke und die Internationalen Ferienkurse in Darmstadt, hrsg. von demselben im Auftrag des Internationalen Musikinstituts Darmstadt (IMD), Saarbrücken 2010, S. 9- 88, insbesondere S. 21-30 und 54-63.

2 Vollständig faksimiliert ebd., S. 29. 
antisemitischen Kampfbund für Deutsche Kultur und dessen Nachfolgeorganisation, der NS-Kulturgemeinde, seine Nähe zum System frühzeitig unter Beweis gestellt. Zum anderen konnte er sich in den entscheidenden Phasen seines beruflichen Aufstiegs der Sympathie des zuständigen kunst- und musikaffinen Ministerialrats Frey sicher sein, was er ihm in dessen Entnazifizierungsverfahren mit einem entlastenden Persilschein dankte. ${ }^{3}$ Ebenfalls hatte Blume die volle Unterstützung aller Rektoren der besonders linientreuen Grenzlanduniversität Kiel, wo er z. B. bereits vor seiner dortigen Berufung im Jahr 1938 als Leiter des Collegium musicum „in den nordschleswigschen und in den völkisch besonders umkämpften Bezirken unserer Provinz ein Zeuge und unmittelbarer Darbieter deutscher Musik“ im „seit langen Jahren geführten Volkstumskampf“4 war. Als entscheidender Beleg seiner politischen Einstellung wurde nach 1938 immer seine musikalische Rassenlehre angeführt, zuletzt in den Akten nachweisbar in einem auf den 9. Juni 1943 datierten Antrag des Dekans der Philosophischen Fakultät auf Übertragung eines Ordinariats für Blume 5 , um dessen Berufung auf die Nachfolge Arnold Scherings zu verhindern, was mit der noch zum 1. September 1944 ausgesprochenen Beförderung auch gelang. Welche Erklärungen außer Gratifikationen für systemkonformes Verhalten kämen daher in Betracht, wenn Blume beispielsweise im Sommer 1939 nach positiver Begutachtung durch Joseph MüllerBlattau auf Kosten der von Herbert Gerigk geführten Hauptstelle Musik der NSDAP im Amt Rosenberg in England Bach- und Händelhandschriften studieren durfte, des Weiteren mit ministerialer Unterstützung zahlreiche Vortragsreisen im Ausland absolvierte und ferner im Jahr 1941 die einmalige Gelegenheit erhielt, gemeinsam mit dem Wiener Kollegen Erich Schenk eine Satzung für einen neu zu gründenden Reichsbund der deutschen Musikwissenschaft auszuarbeiten? ${ }^{6}$

3 Blume nutzte diese Gelegenheit, sich als „bekannter Gegner des Nationalsozialismus“ zu präsentieren, so dass seine Berufung nach Kiel „gegen lebhafte Widerstände im Ministerium“ wiederum seinen damaligen Fürsprecher Frey in günstiges Licht rücken sollte. Blumes Stellungnahme ist auf den 5. August 1947 datiert und erhalten in: Herman-Walther Freys Entnazifizierungsakte Sig. 1BAD461 im Pariser Centre des Archives diplomatiques des Ministère des Affaires étrangères et europénnes.

4 Schreiben des Rektors der Christian-Albrechts-Universität Kiel an Herman-Walther Frey vom 11. Oktober 1937, in: Landesarchiv Schleswig, Akte 47-6469.

5 In: ebd.

6 Dieser Reichsbund wurde vermutlich nie institutionalisiert und ist in der Literatur bislang nicht erwähnt worden, vgl. hierzu vom Autor, „Musikwissenschaftliche Expertennetzwerke vor und nach 1945“, in: ÖMZ 67 (2012), S. 41-47, hier: S. 43 f. 